\title{
The Magnetohydrodynamics Casson Fluid Flow, Heat and Mass Transfer Due to the Presence of Assisting Flow and Buoyancy Ratio Parameters
}

\author{
Shahanaz Parvin ${ }^{1}$, Siti Suzilliana Putri Mohamed Isa ${ }^{1,2,}{ }^{*}$, Norihan Md. Arifin ${ }^{1,3}$, Fadzilah Md Ali ${ }^{1,3}$ \\ Institute for Mathematical Research, Universiti Putra Malaysia, 43400 UPM Serdang, Selangor Darul Ehsan, Malaysia \\ Centre of Foundation Studies for Agricultural Science, Universiti Putra Malaysia, 43400 UPM Serdang, Selangor Darul Ehsan, Malaysia \\ Department of Mathematics, Faculty of Science, Universiti Putra Malaysia, 43400 UPM Serdang, Selangor Darul Ehsan, Malaysia
}

\section{ARTICLE INFO}

\section{Article history:}

Received 21 June 2020

Received in revised form 20 August 2020

Accepted 25 August 2020

Available online 31 August 2020

\section{Keywords:}

Casson fluid; stretching sheet; magnetohydrodynamics; assisting flow; buoyancy ratio

\section{ABSTRACT}

The assisting boundary layer flow, heat and mass transfer have wide applications in engineering devices and in nature: for example, nuclear reactors, heat exchangers, solar receivers, atmospheric flow and lake circulation. Therefore, the numerical study of boundary layer flow, heat and mass transfer on Newtonian or non-Newtonian fluid has to be developed, as a reference to experimental works. Therefore, the mathematical modelling and numerical solutions of boundary layer flow, heat and mass transfer on magneto-hydrodynamics Casson fluid are reported in this paper. The model problem is subjected to the presence of mixed convection with assisting flow, together with the buoyant feature. The Casson fluid is assumed to flow over an exponentially stretching sheet, together with the exponential variations of fluid temperature and fluid concentration. The momentum, energy and concentration equations are formed as the controlling equations and written as partial differential equations (PDE). Subsequently, these equations were transformed into the ordinary differential equations (ODE) by using the similarity transformation. Finally, the ODE are solved numerically by bvp4c program in MATLAB software. The graphs of velocity, temperature and concentration profiles and the numerical values of skin friction coefficient, local Nusselt number and local Sherwood number are presented. These results are obtained due to the controlling parameter, namely as magnetic field, assisting flow and buoyancy ratio parameters. As a result, the increment and decrement of the velocity, temperature, concentration, skin friction coefficient, local Nusselt number and local Sherwood number are influenced by magnetic field, assisting flow and buoyancy ratio parameters.

Copyright @ 2020 PENERBIT AKADEMIA BARU - All rights reserved

* Corresponding author.

E-mail address: ctsuzilliana@upm.edu.my (Siti Suzilliana Putri Mohamed Isa) 


\section{Introduction}

Double-diffusive convection occurs in the fluid due to the combination of temperature and concentration gradients, and both of these components diffuse at different rates. This type of in convection is affected by gravity. The existence of double-diffusive convection can be observed the field of oceanography, astrophysics and geology. Both of these gradients are under the impact of gravitational acceleration of the earth. Therefore, the significant developments of double diffusive convection have been described in details [1-2]. Some of researchers considered the fluid bounded by square cavity [3-5] to explain the double diffusive natural convection. Beside this, Chen and Liu [6] dealed with heated cylinder submerged in a salt-stratified fluid and Kalla et al., [7] considered the case of shallow porous cavity with vertical fluxes of heat and mass in their study. Double diffusive convection in Newtonian fluid [8-11] and various types of non-Newtonian fluid [12-22] are reported with different conditions. These types of non-Newtonian include viscoelastic $[12,17]$, power-law fluid $[13,19]$, nanofluid $[14,20]$, viscoelastic nanofluid [15], Maxwell fluid [16] and Casson fluid [18, 2122].

There are various contributions of boundary layer flow and heat transfer induced by a stretching sheet, to the engineering processes. One of the application is the production of glass fibre, paper and polymer sheet. Therefore, the study of the boundary layer flow, heat and mass transfer bounded by stretching/shrinking sheet are conducted [8-12, 15, 17, 20]. Moreover, Soret and Dufour effects must not be neglected in the process of boundary layer fluid flow with the occurrence of coupled heat and mass transfer. Mass transfer induced by temperature differences is known as Soret effect, whereas Dufour effect refers to the heat transfer caused by concentration gradient in the fluid. The research works that include the impacts of Soret-Dufour parameters were reported by the following references [8-13, 17]. Besides, magnetohydrodynamics related to the occurrence of magnetic properties on electrically conducting fluids. The industrial applications of magnetohydrodynamics are for the production of solar energy by fusion process and cooling of nuclear fission reactors. The significant role of magnetohydrodynamics in the properties of fluid flow, heat and mass transfer contributes to the development of the double-diffusive convection model [17-18, 20].

The central focus of the study is to examine the Casson fluid flow, heat and mass transfer in the presence of magnetic field, assisting flow and buoyancy ratio parameters, which is subjected to the exponential variation of stretching sheet. To our best of knowledge, no report has far been published in the literature with this investigation. MATLAB bvp4c programme is used to calculate the numerical data and graph. Velocity, temperature and concentration profiles for different values of mixed convection parameter, magnetic field parameter and buoyancy parameter are shown graphically for both shrinking and stretching cases. Also, effect of skin friction coefficient, local Nusselt number and local Sherwood number for the above parameter are presented by table. This study is chronologically structured as Methodology, Results and Discussion, and finally Conclusion.

\section{Methodology}

Consider the mathematical formulation for two-dimensional incompressible, viscous and electrically conducting fluid over an exponentially permeable stretching sheet with the effect of magnetic field. The $x$-axis runs along the stretching surface and the $y$-axis is perpendicular to it. The subscripts $w$ and $\infty$ at parameters $T$ and $C$ represent the situation at the stretching sheet and at the point far from the sheet. A transverse magnetic field is assumed to be applied in the $y$-axis and constant magnetic field is denoted by $B_{0}$. As we can see, the temperature and concentration gradients between the point at stretching sheet and at the point out from the fluid region are placed 
in Eq. (2). These components denoted the existence of double-diffusion convection, in magnetohydrodynamics Casson fluid (since the Casson parameter $\omega$ and magnetic field $B_{0}$ also placed in the same equation. The mathematical model of this problem are governed by continuity, momentum, energy and concentration equations Eqs. (1-4). These controlling equations are listed as below:

$\frac{\partial u}{\partial x}+\frac{\partial v}{\partial y}=0$

$\frac{\partial u}{\partial t}+u \frac{\partial u}{\partial x}+v \frac{\partial u}{\partial y}=v\left(1+\frac{1}{\omega}\right) \frac{\partial^{2} u}{\partial y^{2}}+g \beta_{T}\left(T-T_{\infty}\right)+g \beta_{C}\left(C-C_{\infty}\right)+\frac{\sigma B_{0}^{2}}{\rho} u$

$u \frac{\partial T}{\partial x}+v \frac{\partial T}{\partial y}=\alpha \frac{\partial^{2} T}{\partial y^{2}}+\frac{D K_{T}}{C_{S} C_{P}} \frac{\partial^{2} C}{\partial y^{2}}$

$u \frac{\partial C}{\partial x}+v \frac{\partial C}{\partial y}=D \frac{\partial^{2} C}{\partial y^{2}}+\frac{D K_{T}}{T_{m}} \frac{\partial^{2} T}{\partial y^{2}}$

where $u$ and $v$ are the components of velocity in the $x$ and $y$-directions, $v=\frac{\mu}{\rho}$ is the kinematic viscosity, $\mu$ denotes the viscosity, $\rho$ is the fluid density, $g$ is the gravitational acceleration, $\beta_{T}$ is the coefficient of thermal expansion, $\beta_{C}$ is the coefficient of solutal expansions, $T$ is the temperature of the fluid, $C$ is concentration of the fluid, $\sigma$ is the electrical conductivity, $\alpha$ is the thermal diffusivity, $D$ is the solutal diffusivity of the medium, $K_{T}$ is the thermal diffusion ratio, $C_{S}$ is the concentration susceptibility, $C_{P}$ is the specific heat at constant pressure and $T_{m}$ is the mean fluid temperature.

The appropriate boundary conditions are:

$$
\begin{array}{ll}
u=u_{w}(x)=\lambda U_{0} \exp (x / L), v=v_{w}(x), \quad T_{w}(x)=T_{\infty}+T_{0} \exp (x / 2 L), & \text { at } y=0 \\
C_{w}(x)=C_{\infty}+C_{0} \exp (x / 2 L), & \text { at } y \rightarrow \infty
\end{array}
$$

where $\lambda>0$ is the stretching parameter and the wall mass suction velocity is represented by $v_{w}(x)<$ 0 . The term $\exp (x / 2 L)$ in temperature $T_{w}$ and concentration distribution $C_{w}$ are taken from the previous study [8-11], so that the numerical results will satisfy final boundary condition after substituting similarity variables.

Introducing new similarity variables:

$$
\begin{aligned}
& \theta(\eta)=\frac{T-T_{\infty}}{T_{w}-T_{\infty}}, \varphi(\eta)=\frac{C-C_{\infty}}{C_{w}-C_{\infty}}, \quad \eta=y\left(\frac{U_{0}}{2 v L}\right)^{1 / 2} \exp (x / 2 L), \\
& u=U_{0} \exp (x / L) f^{\prime}(\eta), \\
& v=-\left(\frac{v U_{0}}{2 L}\right)^{1 / 2} \exp (x / 2 L)\left[f(\eta)+\eta f^{\prime}(\eta)\right]
\end{aligned}
$$

where prime indicates the differentiation with respect to $\eta$. 
Substituting Eq. (6) into Eqs. (2 - 5), the new ordinary differential equations and boundary conditions are obtained as below:

$$
\begin{aligned}
& \left(1+\frac{1}{\omega}\right) f^{\prime \prime \prime}+f f^{\prime \prime}-2\left(f^{\prime}\right)^{2}+2 R i\left[\exp \left(\frac{-3 X}{2}\right)\right](\theta+N \varphi)-2 H[\exp (-X)] f^{\prime}=0 \\
& \frac{1}{P r} \theta^{\prime \prime}+f \theta^{\prime}-f^{\prime} \theta+D b \varphi^{\prime \prime}=0 \\
& \frac{1}{S c} \varphi^{\prime \prime}+f \varphi^{\prime}-f^{\prime} \varphi+\operatorname{Sr} \theta^{\prime \prime}=0 \\
& f^{\prime}(\eta)=\lambda, \quad f(\eta)=S, \theta(\eta)=1, \varphi(\eta)=1 \quad \text { at } \eta=0 \\
& f^{\prime}(\eta) \rightarrow 0, \quad \theta(\eta) \rightarrow 0, \quad \varphi(\eta) \rightarrow 0 \quad \text { as } \eta \rightarrow \infty
\end{aligned}
$$

The parameters involved in this problem are mixed convection parameter $R i=G r / R e^{2}$, magnetic field parameter $H=2 \sigma L B_{0}^{2} / \rho U_{0}$, thermal Grashof number $G r=g \beta_{T}\left(T_{0}-T_{\infty}\right) L^{3} / v^{2}$, Reynolds number $\operatorname{Re}=U_{0} L / v$, dimensionless coordinate along the plate parameter $X=x / L$, length of the extended/compressed sheet $L$, buoyancy ratio $N=\beta_{C}\left(C_{0}-C_{\infty}\right) / \beta_{T}\left(T_{0}-T_{\infty}\right)$, Prandtl number $\operatorname{Pr}=v / \alpha$, Schmidt number $S c=v / D$, Soret number $\operatorname{Sr}=D K_{T}\left(T_{0}-T_{\infty}\right) /$ $T_{m} v\left(C_{0}-C_{\infty}\right)$, Dufour number $D b=D K_{T}\left(C_{0}-C_{\infty}\right) / C_{S} C_{P} v\left(T_{0}-T_{\infty}\right)$, and suction parameter is defined as $S=\left(v_{w}(x) / \exp (x / 2 L) \times \sqrt{2 L / v U_{0}}\right)>0$. The opposing flow is when $R i<0$. Otherwise, the positive $R i$ indicates the case of aiding flow.

The physical parameters of skin friction coefficient $C_{f}$, local Nusselt number $N u_{x}$, and local Sherwood number $S h_{x}$, are presented as follow:

$$
C_{f}=\left(\frac{\mu}{\rho U_{0}^{2}}\right)\left(\frac{\partial u}{\partial y}\right), \quad N u_{x}=\left(\frac{L}{T_{w}-T_{\infty}}\right)\left(-\frac{\partial T}{\partial y}\right)_{y=0}, \quad S h_{x}=\left(\frac{L}{C_{w}-C_{\infty}}\right)\left(-\frac{\partial C}{\partial y}\right)_{y=0}
$$

Substituting Eq. (6) into Eq. (11), then we get

$$
\begin{aligned}
& C_{f} \sqrt{2 R e_{x}} \exp \left(\frac{-3 X}{2}\right)=f^{\prime \prime}(0), \quad N u_{x} \sqrt{2 / R e_{x}} \exp \left(\frac{-X}{2}\right)=-\theta^{\prime}(0), \\
& S h_{x} \sqrt{2 / R e_{x}} \exp \left(\frac{-X}{2}\right)=-\varphi^{\prime}(0)
\end{aligned}
$$

The final stage of methodology is by performing numerical calculation on the system of exponentially ordinary differential equation together with the boundary conditions Eq. (10) using MATLAB programming. The results are represented in the form of graphs and tables, showing the effect of the controlling parameters namely as magnetic field, assisting flow and buoyancy ratio.

\section{Results and Discussion}

The system of exponentially ordinary differential equations Eqs. (7) to (9), together with the boundary conditions Eq. (10) are solved numerically using MATLAB programming (namely as MATLAB bvp4c). The accuracy of our numerical method is reached by comparing numerical data obtained, with another numerical method as showed in Table 1. Table 1 shows the comparison of skin friction coefficient $C_{f} \sqrt{2 R e_{x}} \exp \left(\frac{-3 X}{2}\right)$, local Nusselt number $N u_{x} \sqrt{2 / R e_{x}} \exp \left(\frac{-X}{2}\right)$ and local Sherwood 
number $S h_{x} \sqrt{2 / R e_{x}} \exp \left(\frac{-X}{2}\right)$ between current data and with shooting method. This technique is developed in Maple software, by converting the Eqs. (7)-(9) into an initial value problem. The fourthorder Runge-Kutta integration scheme is applied to solve the initial value problem. The values for $f^{\prime \prime}(0),-\theta^{\prime}(0)$ and $-\varphi^{\prime}(0)$ are required to perform the shooting technique, together with the predicted highest boundary layer thickness $\eta$. Generally, both of the methods (Maple and MatLab) have to produce numerical results which satisfy final boundary conditions Eq. (10). As a conclusion, the present values are in good agreement with the different numerical technique. Therefore, the good comparison proves that our bvp4c MATLAB is applicable to use for providing subsequent findings in this paper.

The numerical solutions obtained from this article are dual. They are labelled by the first solution (solid line), and the second solution (second line). However, there is only one solution which is stable and physically reliable. Besides, another solution is unstable and not physically occur in actual state in fluid. Therefore, stability analysis is used to select the most stable solution among multiple solutions. However, we did not perform the calculations of stability due to the restrictions of paper pages. In this article, the stable solution is recognized by the pattern of velocity, temperature and concentration variations against boundary layer thickness. The stable solution always following the conditions at the boundary Eq. (10), with minimal existence of minimum or maximum peaks. In this article, the stable solution is declared as the first one. The MATLAB program declares the uniform variation as first solution, otherwise it will be labelled as the second one. Besides, second solution is denoted as unstable, not physically reliable and rejected.

The velocity, temperature and concentration profiles for different values of $H$ are depicted in Figures 1-3. The values of the fluid velocity at a point near the stretching sheet for the both solutions show that the velocity is the lowest when the value of $H=1$. Velocity profile reduces due to the increment of magnetic parameter. The effect of magnetic parameter is to increase the Lorentz force. Consequently, an augmentation of the Lorentz force opposes the flow and the velocity of the fluid is decreased. The temperature profile for the first solution when the sheet is stretched (Figure 2) presents the improvement by addition rate of magnetic parameter $H$. The rate of the fluid temperature for the first solution in Figure 2 are continuously decrease until they reach the zero value. The rate of concentration for the first solution in Figure 3 is the highest, for the highest $H$. It is clear from Figures 2 and 3 that an increase in the magnetic field increases the thickness of thermal and concentration boundary layer. This means that as the magnetic parameter increases, the temperature and concentration profile also increases. The decrement of the magnitude of velocity rate in the boundary layer, due to the effect of magnetic field induces the increment of the fluid temperature and concentration.

The velocity, temperature and concentration profiles for different values of $R i$ are depicted in Figures 4,5 and 6, respectively. The values of the fluid velocity in Figure 4 at a point near the stretching sheet for the both solutions show that the velocity is the lowest when the value of $R i=$ 0.5 . From Figure 5 when the value of $R i=0.5$, the value of temperature is the highest for the first solution while in second solution the value of temperature is the lowest. When the point near the stretching sheet for the value of $R i=0.5$, the maximum peaks exist for the first solution shown in Figure 6 . The variation of concentration profile against $\eta$ for the second solution (in Figure 6 ) shows a decrement, to reach minimum peak. Further, it increases continuously.

The graph of velocity, temperature and concentration profiles are shown in Figures 7-9. These graphs are drawn for various values of $N$. When $N=4$, the momentum boundary layer thickness is the highest for the both solutions at a point near the stretching sheet (Figure 7). This figure presented the addition in velocity in the first solution, due to the addition of $N$, and for small $\eta$. The variation of temperature profile (Figure 8) are continuously decreased until they reach the limit point. The 
values of the fluid temperature for the second solution show that the temperature is the highest for the lowest value of $N$. The concentration is decreased (Figure 9) by the increment of $N$. and for the first solution. Figure 9 shows that the existences of the positive peaks for the first solution at point near the stretching sheet and the negative peaks exist for the second solution. These negative peaks are increased as the value of $N$ increases.

It is clear from Figures 4 and 7 that velocity distribution is increased for the increment of mixed convection parameter $R i$ and buoyancy ratio parameter $N$. This is due to the fact that higher rate of mixed convection parameter is caused by a greater buoyancy effects. Therefore, the fluid flow is accelerated and the instantaneous velocity increases. Figures 5-6 and 8-9 show that the temperature and concentration decrease with increasing values of mixed convection parameter $R i$ and buoyancy ratio parameter $N$. When parameter $R i$ (i.e. buoyancy effects) increase, it contributes to the increment of the convection cooling effect. As a result, the temperature and concentration reduce.

The tabulation of skin friction coefficient, local Nusselt number and local Sherwood number for the impact of parameters $H, R i$ and $N$ is presented in Table 2. It can be seen from Table 2 that the increment values of $H$ cause the values of skin friction coefficient number, local Nusselt number and Sherwood number become decrease for the first solution. Second solution shows that the values of skin friction coefficient number and local Nusselt number decrease but the values of local Sherwood number increase due to increasing value of $H$. Table 2 also shows that the first solution always had an increment for the skin friction coefficient, the local Nusselt number and the local Sherwood number caused by the effect of increasing parameter $R i$. Moreover, second solution only had an increment in skin friction coefficient and the local Sherwood number which are affected by the same parameter. The effect of parameter $N$ is to increase the values of skin friction coefficient number, local Nusselt number and local Sherwood number for the first solution. However, the impact of parameter $N$ for the second solution is to enhance the values of the skin friction coefficient number and the local Nusselt number. A decrement in the values of the Sherwood number observed in second solution due to an increment in the values of $N$.

\section{Table 1}

The comparison with shooting method for various values of $\omega$ and $H$ when $\omega=100, R i=0.5, S r=2.0$, $\mathrm{Db}=0.03, \mathrm{X}=0.1, \lambda=1, \mathrm{Sc}=1, \mathrm{~N}=0.5, \operatorname{Pr}=1, \mathrm{~S}=2.5$

\begin{tabular}{llllllll}
\hline \multirow{2}{*}{ Solution } & $H$ & \multicolumn{2}{l}{$C_{f} \sqrt{2 R e_{x}} \times \exp \left(\frac{-3 X}{2}\right)$} & \multicolumn{2}{c}{$N u_{x} \sqrt{2 / R e_{x}} \times \exp \left(\frac{-X}{2}\right)$} & \multicolumn{2}{c}{$S h_{x} \sqrt{2 / R e_{x}} \times \exp \left(\frac{-X}{2}\right)$} \\
& & (a) & (b) & (a) & (b) & (a) & (b) \\
\hline \multirow{3}{*}{ First } & 0.5 & -2.68915 & -2.68916 & 2.94868 & 2.94869 & -2.64919 & -2.64918 \\
& 1 & -2.94117 & -2.94117 & 2.93502 & 2.93501 & -2.66278 & -2.66278 \\
& 1.5 & -3.16702 & -3.16701 & 2.92340 & 2.92340 & -2.67369 & -2.67368 \\
& 0.5 & -4.18403 & -4.18403 & 3.10758 & 3.10757 & -2.99277 & -2.99277 \\
Second & 1 & -4.44169 & -4.44168 & 3.14122 & 3.14122 & 0.93555 & 0.93554 \\
& 1.5 & -4.64893 & -4.64893 & 3.44751 & 3.44750 & 3.40391 & 3.40391
\end{tabular}

(a) Current values, (b) Values obtained by shooting method 


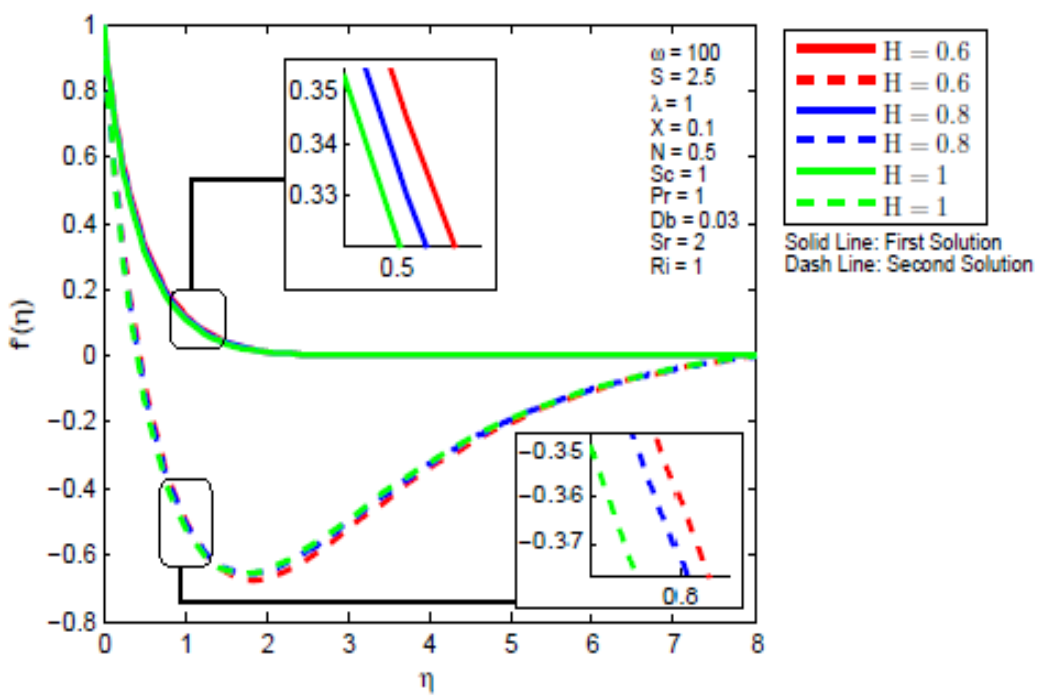

Fig. 1. Velocity profile for different values of $H$

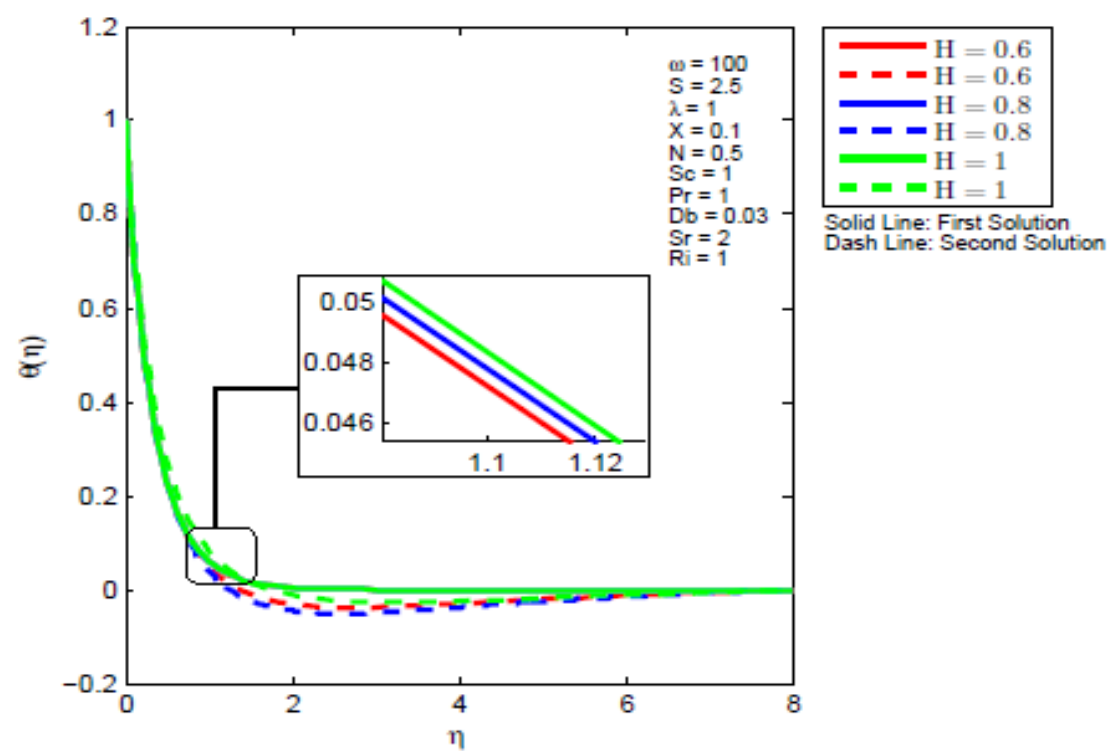

Fig. 2. Temperature profiles for different value of $H$

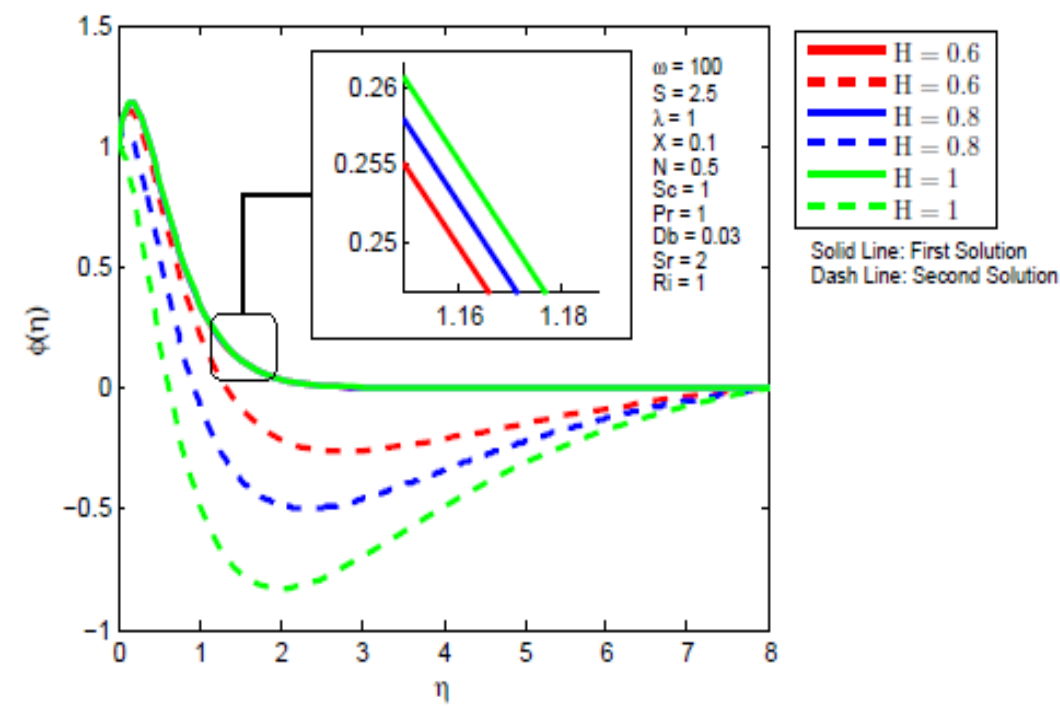

Fig. 3. Concentration profiles for different value of $H$ 


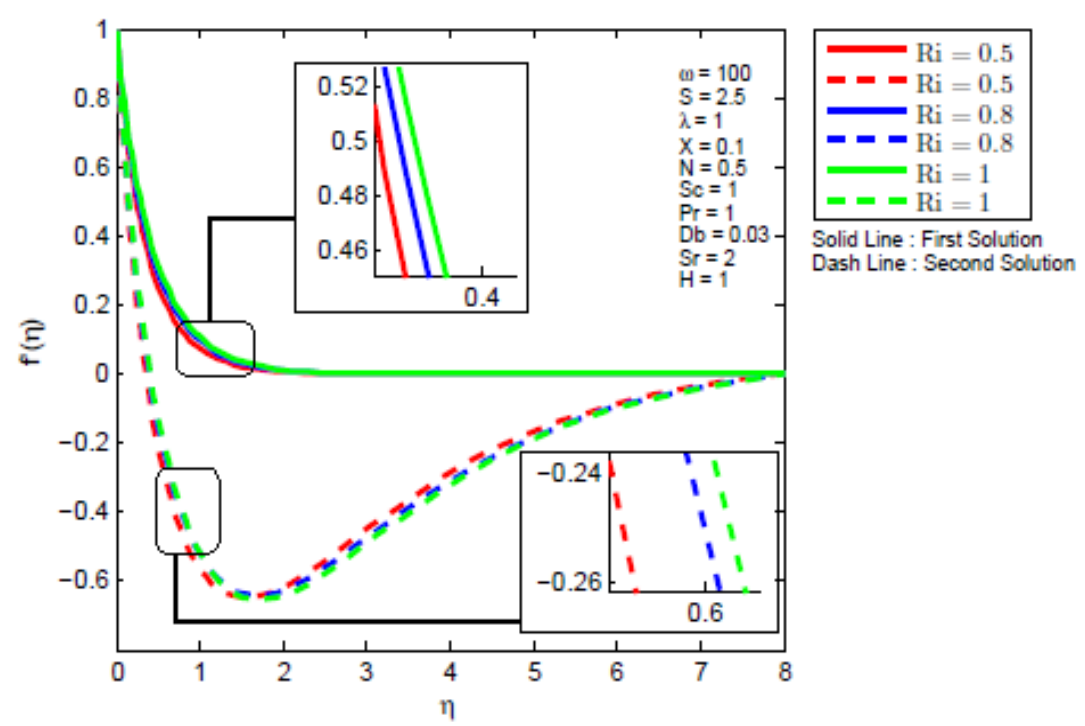

Fig. 4. Velocity profile for different values of $R i$

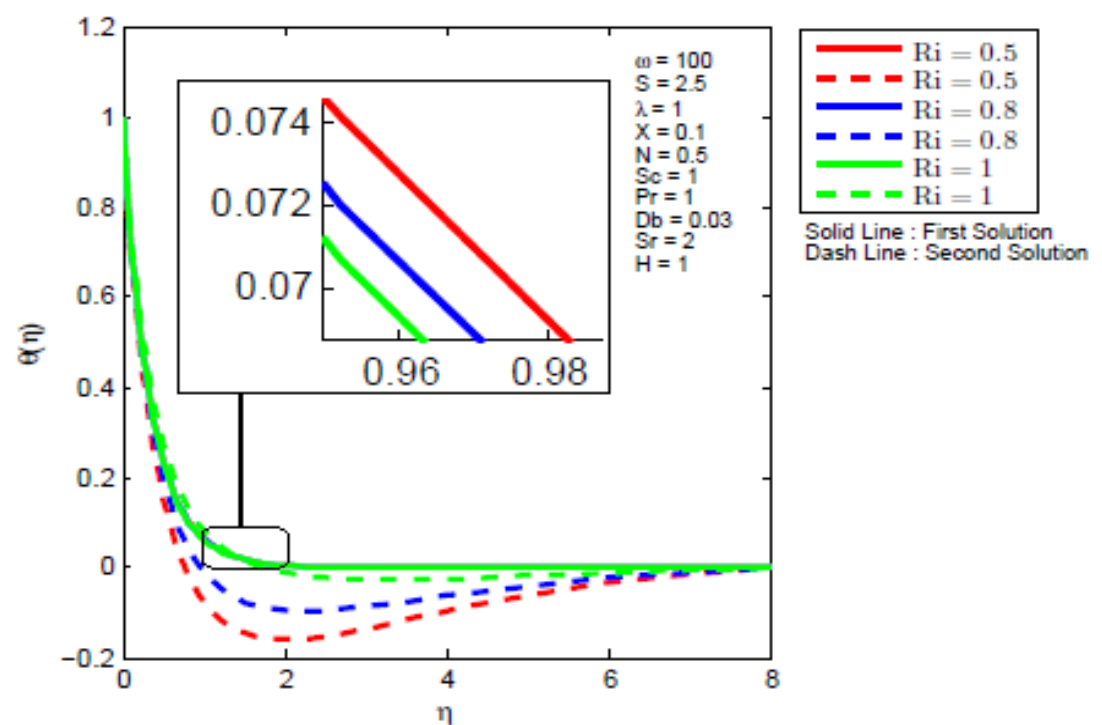

Fig. 5. Temperature profile for different value of $R i$

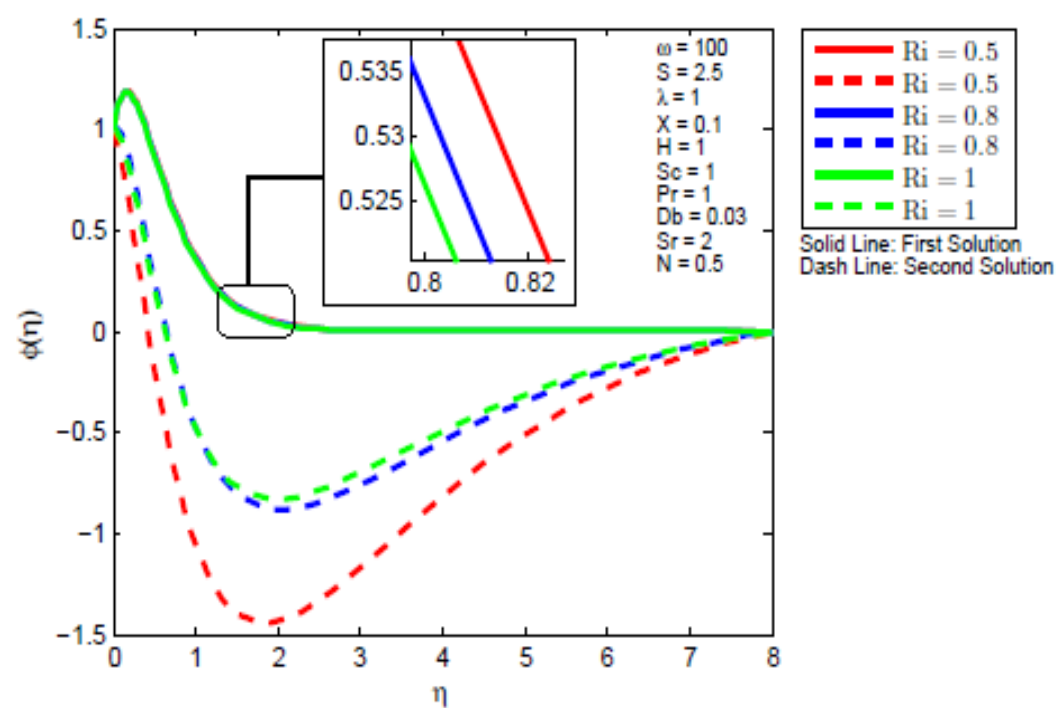

Fig. 6. Concentration profile for different value of $R i$ 


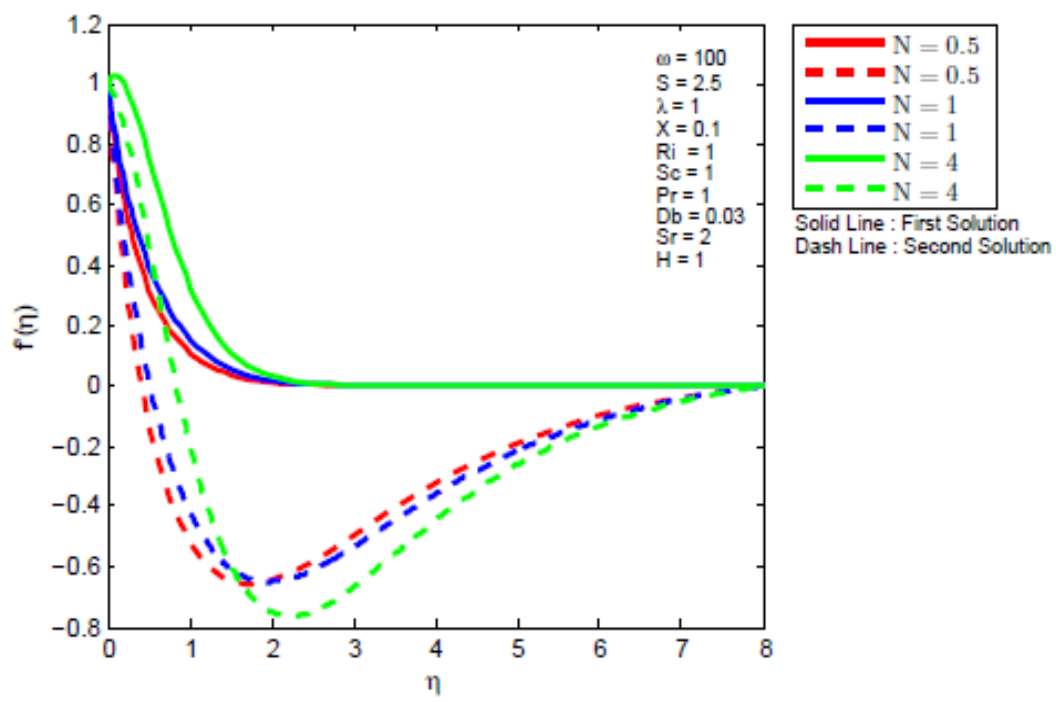

Fig. 7. Velocity profiles for different value of $N$

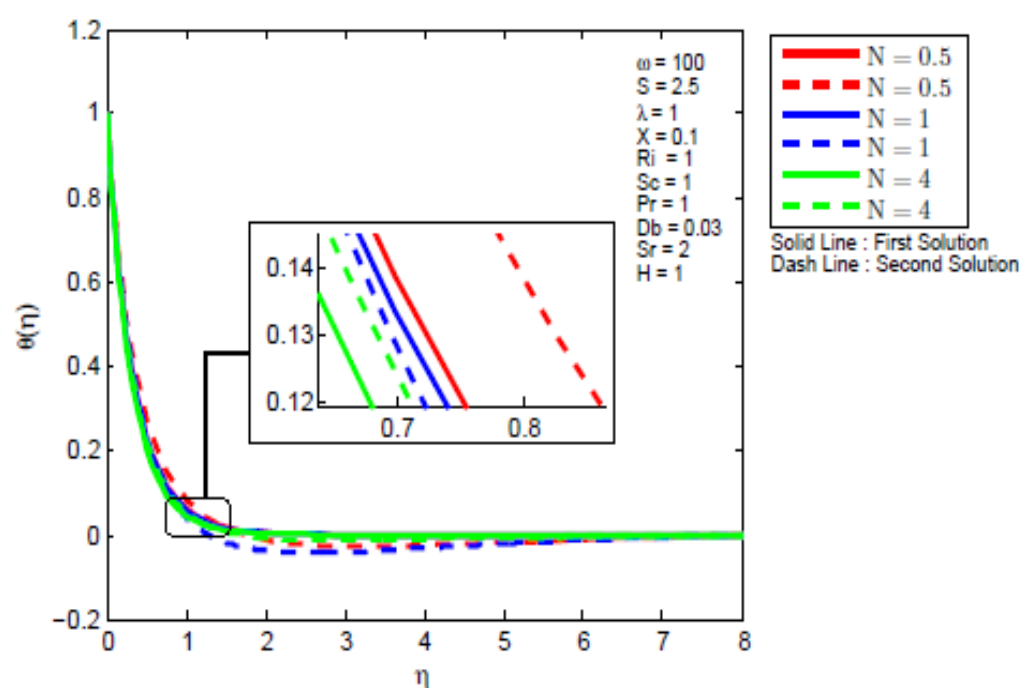

Fig. 8. Temperature profiles for different value of $N$
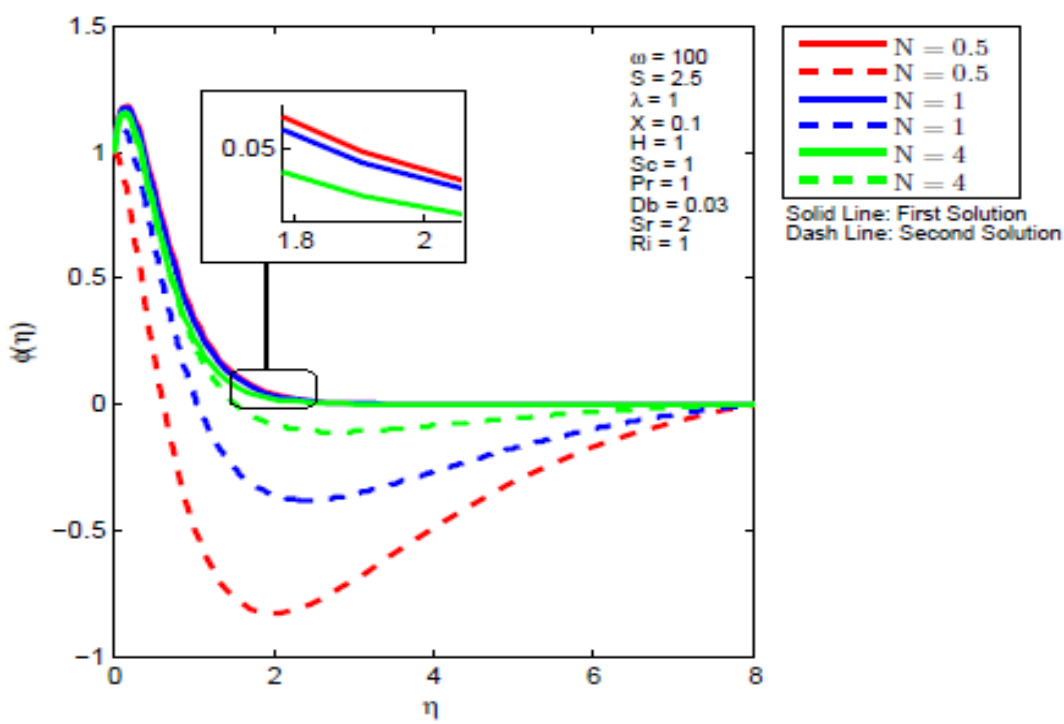

Fig. 9. Concentration profiles for different value of $N$ 


\section{Table 2}

The values of skin friction coefficient, local Nusselt number and local Sherwood number due to the effect of parameters $H, R i$ and $N$

\begin{tabular}{|c|c|c|c|c|c|c|c|}
\hline Parameters & Values & $\begin{array}{l}\quad C_{f} \sqrt{2 R} \\
\text { First } \\
\text { solution }\end{array}$ & $\begin{array}{l}\exp \left(\frac{-3 X}{2}\right) \\
\text { Second } \\
\text { solution }\end{array}$ & $\begin{array}{l}N u_{x} \sqrt{2 /} \\
\text { First } \\
\text { solution }\end{array}$ & $\begin{array}{l}\times \exp \left(\frac{-X}{2}\right) \\
\text { Second } \\
\text { solution }\end{array}$ & $\begin{array}{l}S h_{x} \sqrt{2 / R e_{x}} \\
\text { First } \\
\text { solution }\end{array}$ & $\begin{array}{c}\times \exp \left(\frac{-X}{2}\right) \\
\text { Second } \\
\text { solution } \\
\end{array}$ \\
\hline \multirow{3}{*}{$H$} & 0.6 & -2.24482 & -3.55708 & 2.97580 & 2.89334 & -2.61965 & -2.31887 \\
\hline & 0.8 & -2.35792 & -3.70017 & 2.96950 & 2.90050 & -2.62615 & -1.48953 \\
\hline & 1 & -2.46571 & -3.88426 & 2.96361 & 2.73122 & -2.63212 & -0.04634 \\
\hline \multirow{3}{*}{$R i$} & 0.5 & -2.94117 & -4.44169 & 2.93502 & 3.14122 & -2.66278 & 0.93555 \\
\hline & 0.8 & -2.65316 & -4.05712 & 2.95254 & 3.00205 & -2.64382 & -0.49706 \\
\hline & 1 & -2.46571 & -3.88426 & 2.96361 & 2.73122 & -2.63212 & -0.04634 \\
\hline \multirow{3}{*}{$N$} & 0.5 & -2.46571 & -3.88426 & 2.96361 & 2.73122 & -2.63212 & -0.04634 \\
\hline & 1 & -1.97500 & -3.17300 & 2.99533 & 2.93917 & -2.59654 & -1.83899 \\
\hline & 4 & 0.56325 & -0.28188 & 3.13401 & 3.05900 & -2.45916 & -2.38784 \\
\hline
\end{tabular}

\section{Conclusions}

The magnetohydrodynamics casson fluid flow, heat and mass transfer in the presence of assisting flow and buoyancy ratio parameters are reported. From the graphs and tables, following conclusion are listed as below:

I. Fluid velocity of the first solution is enhanced due to the increment of mixed convection and buoyancy ratio parameters. In addition, minimum peak arises in the velocity graphs for the small $\eta$ on the second solution.

II. The impact of magnetic field parameter is to increase the rate of fluid temperature and concentration of the first solution. The temperature and concentration is highest at the wall $(\eta=0)$.

III. The increment values of skin friction coefficient, local Nusselt number and local Sherwood number on the first solution are influenced by the augmentation of parameters $R i$ and $N$.

\section{Acknowledgement}

This research was funded by a grant from Universiti Putra Malaysia (Project code: GPIPM/2018/9596900).

\section{References}

[1] Huppert, H. E., and D. R. Moore. "Nonlinear double-diffusive convection." Journal of Fluid Mechanics 78, no. 4 (1976): 821-854.

[2] Huppert, H. E., and J. S. Turner. "Double-diffusive convection." Journal of Fluid Mechanics 106 (1981): $299-329$.

[3] Beghein, C., F. Haghighat, and F. Allard. "Numerical study of double-diffusive natural convection in a square cavity." International Journal of Heat and Mass Transfer 35, no. 4 (1992): 833-846. https://doi.org/10.1016/0017-9310(92)90251-M

[4] Costa, V. A. F. "Double diffusive natural convection in a square enclosure with heat and mass diffusive walls." International Journal of Heat and Mass Transfer 40, no. 17 (1997): 4061-4071.

https://doi.org/10.1016/S0017-9310(97)00061-6

[5] Badruddin, I. A., T. M. Y. Khan, N. J. S. Ahmed, and S. Kamangar. "Effect of variable heating on double diffusive flow in a square porous cavity." In AIP Conference Proceedings, vol. 1728, no. 1, p. 020689. AIP Publishing, 2016. https://doi.org/10.1063/1.4946740

[6] Chen, Y. M., and C. K. Liu. "Double-diffusive convection for a heated cylinder submerged in a salt-stratified fluid layer." Heat and mass transfer 33, no. 1-2 (1997): 17-26.

https://doi.org/10.1007/s002310050157 
[7] Kalla, L., M. Mamou, P. Vasseur, and L. Robillard. "Multiple solutions for double diffusive convection in a shallow porous cavity with vertical fluxes of heat and mass." International journal of heat and mass transfer 44, no. 23 (2001): 4493-4504. https://doi.org/10.1016/S0017-9310(01)00076-X

[8] Isa, S. S. P. M., N. M. Arifin, and U. Farooq. "Effect of Soret and Dufour numbers on double diffusive mixed convection boundary layer flow induced by a shrinking sheet." Journal of Physics: Conference Series 1298, (2019): 012024.

https://doi.org/10.1088/1742-6596/1298/1/012024

[9] Srinivasacharya, D., and Ch RamReddy. "Soret and Dufour effects on mixed convection from an exponentially stretching surface." International Journal of Nonlinear Science 12, no. 1 (2011): 60-68.

[10] Sreenivasulu, P., and N. Bhaskar Reddy. "Thermo-diffusion and diffusion-thermo effects on MHD boundary layer flow past an exponential stretching sheet with thermal radiation and viscous dissipation." Advances in Applied Science Research 3, no. 6 (2012): 3890-3901.

[11] Hayat, T., T. Muhammad, S. A. Shehzad, and A. Alsaedi, "Soret and Dufour effects in three-dimensional flow over an exponentially stretching surface with porous medium, chemical reaction and heat source/sink." International Journal of Numerical Methods for Heat \& Fluid Flow 25, no. (2015): 762-781. https://doi.org/10.1108/HFF-05-2014-0137

[12] Gbadeyan, J. A., A. S. Idowu, A. W. Ogunsola, O. O. Agboola, and P. O. Olanrewaju. "Heat and mass transfer for Soret and Dufour's effect on mixed convection boundary layer flow over a stretching vertical surface in a porous medium filled with a viscoelastic fluid in the presence of magnetic field." Global Journal of Science Frontier Research 11, no. 8 (2011): 97-114.

[13] Srinivasacharya, D., and S. Reddy. "Double diffusive natural convection in power-law fluid saturated porous medium with Soret and Dufour effects." Journal of the Brazilian Society of Mechanical Sciences and Engineering 34, no.4 (2012): 525-530 https://doi.org/10.1590/S1678-58782012000400014.

[14] Murthy, P. V. S. N., A. Sutradhar, and Ch RamReddy. "Double-diffusive free convection flow past an inclined plate embedded in a non-Darcy porous medium saturated with a nanofluid." Transport in Porous Media 98, no.3 (2013):553-564.

https://doi.org/10.1007/s11242-013-0160-z

[15] Goyal, M., and R. Bhargava. "Finite element solution of double-diffusive boundary layer flow of viscoelastic nanofluids over a stretching sheet." Computational Mathematics and Mathematical Physics 54, no. 5 (2014): 848863. https://doi.org/10.1134/S0965542514050029

[16] Hayat, T., M. Farooq, and A. Alsaedi. "Melting heat transfer in the stagnation-point flow of Maxwell fluid with double-diffusive convection." International Journal of Numerical Methods for Heat and Fluid Flow 24, no. 3 (2014): 760-774.

https://doi.org/10.1108/HFF-09-2012-0219

[17] Rashidi, M. M., M. Ali, B. Rostami, P. Rostami, and G. N. Xie. "Heat and mass transfer for MHD viscoelastic fluid flow over a vertical stretching sheet with considering Soret and Dufour effects." Mathematical Problems in Engineering 2015 (2015): 861065. https://doi.org/10.1155/2015/861065

[18] Raju, C. S. K., and N. Sandeep. "Heat and mass transfer in MHD non-Newtonian bio-convection flow over a rotating cone/plate with cross diffusion." Journal of Molecular Liquids 215 (2016): 115-126. https://doi.org/10.1016/j.molliq.2015.12.058

[19] Zhuang, Y. J., H. Z. Yu, Q. Y. Zhu. "A thermal non-equilibrium model for 3D double diffusive convection of powerlaw fluids with chemical reaction in the porous medium." International Journal of Heat and Mass Transfer 115, Part B, (2017): 670-694 https://doi.org/10.1016/i.ijheatmasstransfer.2017.08.068

[20] Pal, D., and G. Mandal. "Double diffusive magnetohydrodynamic heat and mass transfer of nanofluids over a nonlinear stretching/shrinking sheet with viscous-Ohmic dissipation and thermal radiation." Propulsion and Power Research 6, no. 1 (2017): 58-69. https://doi.org/10.1016/j.jppr.2017.01.003

[21] Prasad, D.V. K., G. S. K. Chaitanya, R. S. Raju. "Double diffusive effects on mixed convection Casson fluid flow past a wavy inclined plate in presence of Darcian porous medium." Results in Engineering 3, (2019): 100019 https://doi.org/10.1016/i.rineng.2019.100019 
[22] Alkasasbeh, Hamzeh Taha. "Numerical solution of micropolar Casson fluid behaviour on steady MHD natural convective flow about a solid sphere." Journal of Advanced Research in Fluid Mechanics and Thermal Sciences 50, no. 1 (2018): 55-66. 